\title{
Clinical Effects of Pantoprazole and Octreotide in Upper Gastrointestinal Hemorrhage
}

\author{
Yong Wang', Fei Deng ${ }^{2 *}$ \\ ${ }^{1}$ Department of Gastroenterology, Hanchuan People's Hospital, Xiaogan, Hubei, CHINA. \\ ${ }^{2}$ Department of Respiratory Medicine, Hanchuan People's Hospital, Xiaogan, Hubei, CHINA.
}

\begin{abstract}
Objective: This study aimed to discuss the clinical effects of pantoprazole and octreotide in upper gastrointestinal hemorrhage. Methods: A total of 114 patients with upper gastrointestinal hemorrhage admitted in our hospital from April 2016 to June 2017 were chosen and divided into control and experimental groups by lottery method. The control group $(n=57)$ was treated with pantoprazole and the experimental group $(n=57)$ was treated with pantoprazole + octreotide. The therapeutic effect, occurrence rate of untoward effects, hemostatic effects at 24 and $72 \mathrm{~h}$, blood-transfusion volume, hemostatic time and rehemorrhage rate at $48 \mathrm{~h}$ of the two groups were compared. Results: Compared with the control group, the experimental group exhibited significantly higher total therapeutic and hemostatic effects at $24 \mathrm{~h}$, as well as lower occurrence rate of untoward effects and hemostatic effects at $24 \mathrm{~h}$. Statistically significant differences were observed between the control and experimental groups $(P<0.05)$. The experimental group had greater blood-transfusion volume, shorter hemostatic time and higher rehemorrhage rate at $48 \mathrm{~h}(P<0.05)$. Conclusion: The pantoprazole + octreotide therapy exerted outstanding clinical effects on upper gastrointestinal hemorrhage. This combined therapy can decrease the occurrence rate of untoward effects and bloodtransfusion volume, reduce hemostatic time and prevent recurrent bleeding, thereby being worthy of clinical use and promotion.
\end{abstract}

Key words: Octreotide, Pantoprazole, Upper Gastrointestinal hemorrhage

\section{INTRODUCTION}

Upper gastrointestinal hemorrhage is a common emergency condition in clinical medicine. This condition can attack all age groups and it has a high occurrence rate of complications and mortality. ${ }^{1}$ Upper gastrointestinal hemorrhage is the joint consequence of multiple complex and varying factors. Under normal conditions, gastric hemorrhage, acute hemorrhagic gastritis, Mallory-Weiss syndrome and peptic ulcer are main causes of upper gastrointestinal hemorrhage. ${ }^{2}$ Effective treatment lies in the timely fast hemostasis, reasonable selection of good hemostatic drugs and fast bleeding control. At present, conservative medication is the preferred choice of clinical therapy. ${ }^{3}$ Thus, reasonable and effective ways of using medicines and treatments must be established to reduce mortality and improve the prog- nosis and living quality of patients. To evaluate the clinical effects and safety of the pantoprazole + octreotide combined therapy in upper gastrointestinal hemorrhage, 114 patients with upper gastrointestinal hemorrhage admitted in our hospital from April 2016 to June 2017 were chosen and divided into the control and experimental groups. The results are discussed in the following text.

\section{MATERIALS AND METHODS}

A total of 114 patients with upper gastrointestinal hemorrhage admitted in our hospital from April 2016 to June 2017 were chosen. The inclusion standards were: This study was approved by the Ethics Commit-

\section{General information}

Submission Date: 22-06-2018; Revision Date: 14-08-2018; Accepted Date: 29-12-2018

DOI: 10.5530/ijper.53.2.42 Correspondence: Dr. Fei Deng,

Department of Respiratory Medicine, Hanchuan

People's Hospital, Xiaogan, Hubei, CHINA.

Phone: +86-0712-8282259 E-mail: fei_deng666@126. com

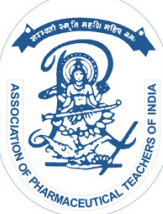

www.ijper.org 
tee of our hospital. All patients signed Informed Consent forms after being informed about the goal of the study. The exclusion standards were: pregnant women and women in suckling period; patients with malignant tumors and blood diseases; patients with abnormal liver, kidney, heart and brain functions or with metal diseases; and patients with drug contraindications or drug allergy history. The patients were divided into the control and experimental groups through lottery method. The control group $(n=57)$ consisted of 28 males and 29 females who had an age range of 34-78 years old (average: 56.5 \pm 5.3). The control group included 15 cases of esophageal varices, eight cases of gastric cancer, eight cases of acute erosive gastritis, 20 cases of gastric ulcer and six cases of intestinal ulcer. The experimental group $(n=57)$ consisted of 30 males and 27 females who had an age range of 35-79 years old (average: 57.3 \pm 5.2 ). The experimental group included 17 cases of esophageal varices, 10 cases of gastric cancer, 10 cases of acute erosive gastritis, 12 cases of gastric ulcer and eight cases of intestinal ulcer. Statistical analysis indicated that the two groups were significantly different in general information $(P>0.05)$, indicating that these two groups were comparable.

\section{Methods}

Basic treatment was conducted as follows. After being admitted to the hospital, the patients were kept lying down under another person's assistance. The patients were forbidden to eat and oxygen uptake was offered immediately. Symptomatic treatment, including timing blood transfusion and transfusion, was implemented. In addition, nutrient supports were given and the protopathy was treated positively. At the same time, the blood pressure, heart rate, respiration, pulse, gastric tube drainage fluid and the fecal color and frequency of the patients were monitored closely. Stool routine examination, blood routine examination and hepatorenal function examination were performed regularly. The control group was treated with pantoprazole (SFDA approval number: H19990166, Hangzhou Sino-American Pharmaceutical Co., Ltd., specification: chemical drug, $40 \mathrm{mg}$ ). An intravenous drip of $40 \mathrm{mg}$ pantoprazole $+100 \mathrm{~mL}$ $0.9 \%$ sodium chloride solution was given twice per day. Medication to patients with gastric cancer and renal insufficiency must be considered carefully. The experimental group was given octreotide (SFDA approval number: H20090291, Jiangsu Osekang Pharmaceutical Co., Ltd., specification: chemical drug, $0.1 \mathrm{mg}$ (counted at octreotide)) according to the treatment to the control group. An amount of $0.2 \mathrm{mg}$ octreotide+20 mL 0.9\% sodium chloride solution was intravenously pumped at $25-25 \mu \mathrm{g} / \mathrm{h}$. The pumping rate was adjusted according to the amount of bleeding. This treatment was given continuously for three days. Blood transfusion was offered to patients with hemoglobin count of $\leq 70 \mathrm{~g} / \mathrm{L}$.

\section{Observation indices and evaluation of therapeutic effect}

First, the hemostatic effects at 24 and $72 \mathrm{~h}$ of the two groups were evaluated according to following standards. The clinical symptoms were improved to some extent. The blood pressure and pulse recovered to the normal state. The gastric tube drainage fluid was cool. Gastroscopy revealed that bleeding had stopped completely and new fresh bleeding signs were observed on the ulcer surface. Hematemesis disappeared and feces turned yellow. Occult blood test was negative and hemoglobin did not decline continuously. Effective hemostasis occurred if any one of these three standards was met and hemostasis failure occurred if none was met.

Second, the untoward effects in the two groups during the treatment were observed and recorded. Common untoward effects mainly included loss of appetite, dizziness, vomiting, retching, thirst, etc.

Third, the hemostatic time, blood-transfusion volume and rehemorrhage rate at $48 \mathrm{~h}$ were recorded.

Fourth and last, therapeutic effect was classified according to the following evaluation standards: significantly effective: after 1 day of treatment, hemorrhage symptoms disappeared, systolic pressure $(>90 \mathrm{mmHg})$ and diastolic pressure $(>60 \mathrm{mmHg})$ recovered to normal standards and pulse $>60$ times/min; effective: after 1-3 days of treatment, hemorrhage symptoms disappeared, blood pressure and pulse recovered to the normal standards; and ineffective: live hemorrhage symptoms still existed after 3 days of treatment, blood pressure and pulse fluctuated violently. Total therapeutic effect $=$ significantly effective + effective.

\section{Statistical analysis}

The data were analyzed by using SPSS 21.0. The measurement data (e.g., hemostatic time and blood-transfusion volume) were expressed as $\overline{\mathrm{x}} \pm s$ and examined by conducting t-test. The enumeration data (e.g., total therapeutic effect, occurrence rate of untoward effects and hemostatic effects) were expressed in \% and examined by using $\mathrm{X}^{2}$-test. $P<0.05$ indicated a statistically significant difference. 


\section{RESULTS}

\section{Therapeutic effects}

The experimental group presented significantly higher total therapeutic effects than that of the control group $(P<0.05)$. The results are shown in Table 1.

\section{Hemostatic effects}

Compared with the control group, the experimental group showed higher hemostatic effect at $24 \mathrm{~h}$ but lower hemostatic effect at $72 \mathrm{~h}(P<0.05)$. The results are shown in Table 2.

\section{Blood-transfusion volume, hemostatic time and rehemorrhage rate at $48 \mathrm{~h}$}

The experimental group showed significantly lower blood-transfusion volume, hemostatic time and rehemorrhage rate at $48 \mathrm{~h}$ than those of the control group $(P<0.05)$. The results are shown in Table 3.

\section{Occurrence rate of untoward effects}

The occurrence rate of untoward effects of the control group was $22.81 \%$, which is significantly higher than that of the experimental group $(7.02 \%)(P<0.05)$. The results are shown in Table 4.

\section{DISCUSSION}

Upper gastrointestinal hemorrhage is a common critical disease in clinics. Its major symptoms include dizziness, hematemesis, melena and cold sweet. ${ }^{4}$ This condition is characterized by the sudden onset, fast development, high mortality and serious threats to the life safety and physical health of patients. At present, external materials, surgical bypass and endoscope are the main clinical treatment procedures for upper gastrointestinal hemorrhage. ${ }^{5}$ Among them, conservative medication is still the preferred choice. Inhibitors are common hemostatic drugs for non-varicosity upper gastrointestinal

\begin{tabular}{|c|c|c|c|c|}
\hline \multicolumn{5}{|c|}{ Table 1: Comparison of the therapeutic effects between two groups [n (\%)]. } \\
\hline Groups & Significantly effective & Effective & Ineffective & Total \\
\hline Experimental $(n=57)$ & $28(49.12)$ & $26(45.61)$ & $3(5.26)$ & $54(94.74)$ \\
\hline Control $(n=57)$ & $24(42.11)$ & $22(38.59)$ & $11(19.29)$ & $46(80.70)$ \\
\hline$X^{2}$ & & & & 5.2114 \\
\hline$P$ & & & & 0.0224 \\
\hline
\end{tabular}

\begin{tabular}{|c|c|c|}
\hline \multicolumn{2}{|c|}{ Table 2: Comparison of hemostatic effects between the two groups [n (\%)]. } \\
\hline Groups & Hemostatic effect at 24h & Hemostatic effect at 72h \\
\hline Experimental $(n=57)$ & $36(63.16)$ & $21(36.84)$ \\
\hline Control $(n=57)$ & $23(40.35)$ & 5.9371 \\
\hline$X^{2}$ & & 0.0148 \\
\hline$P$ & & $59.65)$ \\
\hline
\end{tabular}

Table 3: Comparison of the blood-transfusion volume, hemostatic time and rehemorrhage rate at $48 \mathrm{~h}$ between the two groups $(x \pm s)$.

\begin{tabular}{|c|c|c|c|}
\hline Groups & Blood-transfusion volume $(\mathbf{U})$ & Hemostatic time $(\mathbf{h})$ & Rehemorrhage rate at 48h $(\%)$ \\
\hline Experimental $(n=57)$ & $1.12 \pm 0.08$ & $17.92 \pm 2.78$ & $1(1.75)$ \\
\hline Control $(n=57)$ & $2.32 \pm 0.10$ & $30.12 \pm 3.20$ & $7(12.28)$ \\
\hline$t^{2}$ & 70.7451 & 21.7291 & 4.8396 \\
\hline$P$ & 0.0000 & 0.0000 & 0.0278 \\
\hline
\end{tabular}

\begin{tabular}{|c|c|c|c|c|c|}
\hline \multicolumn{2}{|c|}{ Table 4: Comparison of the occurrence rate of untoward effects between the two groups [n (\%)]. } \\
\hline Groups & Loss of appetite & Vomiting and retching & Thirst & Dizzy & Untoward effects \\
\hline Experimental $(n=57)$ & $1(1.75)$ & $1(1.75)$ & $2(3.51)$ & $0(0.00)$ & $4(7.02)$ \\
\hline Control $(n=57)$ & $4(7.02)$ & $2(3.51)$ & $5(8.77)$ & $2(3.51)$ & $13(22.81)$ \\
\hline$X^{2}$ & & & & & 4.6531 \\
\hline$P$ & & & & & 0.0310 \\
\hline
\end{tabular}


hemorrhage. It can reduce pepsin activity, accelerate thrombus freezing and prevent the proease damage, thus enabling to reach the expected hemostatic effects. ${ }^{6,7}$ However, the use of acid inhibitors alone cannot achieve satisfying hemostatic effects.

Pantoprazole is a third-generation proton-pump inhibitor with strong target specificity and acid stability. It offers non-competitive selective inhibition of the proton pump $\mathrm{H}^{+}-\mathrm{K}^{+}$-ATP enzyme in the cell membrane and interdict the final step of gastric acid secretion, thus effectively inhibiting gastric acid secretion and realizing hemostasis. ${ }^{8,9}$ As an octapeptide ring compound, octreotide is mainly synthesized artificially and it can develop the therapeutic effect of natural endogenous somatostatin. Octreotide is characterized by having strong effect and long duration. The half-life period of octreotide is more than 30 times that of natural demodicidin. ${ }^{10}$ Octreotide can induces direct selective effects on vascular smooth muscles; significantly reduce the visceral blood flow; effectively inhibit the secretions of gastric acid, gastrin and protease; stimulate mucous secretion; protect the mucosal barrier; and recover mucous epithelial cells. ${ }^{11-12}$ Moreover, octreotide can increase the lower sphincter tension of the large esophagus, improve the gastric juice reflux, protect the esophagus mucosa and promote blood clot retraction and platelet aggregation. Octreotide offers outstanding therapeutic effects in alimentary tract hemorrhage, acute pancreatitis, intestinal obstruction and many other diseases.

\section{CONCLUSION}

In this study, significant differences were observed between the experimental and control groups in the total therapeutic effect, hemostatic effect at $24 \mathrm{~h}$, occurrence rate of untoward effects and hemostatic effect at $72 \mathrm{~h}$ $(P<0.05)$. The experimental group was superior to the control group in blood-transfusion volume, hemostatic time and rehemorrhage rate at $48 \mathrm{~h}(P<0.05)$. These findings proved that the pantoprazole + octreotide combined therapy presented outstanding clinical effects in upper gastrointestinal hemorrhage. The proposed treatment can reduce the occurrence rate of untoward effects and blood-transfusion volume, reduce hemostatic time and prevent recurrent bleeding. As such, this strategy is worthy of clinical use and promotion.

\section{ACKNOWLEDGEMENT}

This is no financial support for this work, so it does not need the acknowledge.

\section{CONFLICT OF INTEREST}

The authors declare no conflict of interest.

\section{ABBREVIATIONS}

SFDA: State Food and Drug Administration.

\section{SUMMARY}

Upper gastrointestinal hemorrhage is a common emergency condition in clinical medicine. Hence it is necessary to develop effective ways of using medicines and treatments to reduce mortality and improve the prognosis and living quality of patients. In this study, we show that the pantoprazole+octreotide therapy can exert outstanding clinical effects on upper gastrointestinal hemorrhage. This combined therapy can decrease the occurrence rate of untoward effects and blood-transfusion volume, reduce hemostatic time and prevent recurrent bleeding, thereby being worthy of clinical use and promotion.

\section{REFERENCES}

1. Trawick EP, Yachimski PS. Management of non-variceal upper gastrointestinal tract hemorrhage: Controversies and areas of uncertainty. World J Gastroenterol. 2012;18(11):1159-65.

2. Lu DB, Wang XY, Wang J. Inhibition of Human Carboxylesterases (CES) by Gastrointestinal Tumors Medication Trametinib and Regorafenib. Lat Am J Pharm. 2017;36(10):1936-8.

3. Stanley AJ. Update on risk scoring systems for patients with upper gastrointestinal haemorrhage. World J Gastroenterol. 2012;18(22):2739-44.

4. Crooks C, Card T, West J. Reductions in 28-Day Mortality Following Hospital Admission for Upper Gastrointestinal Hemorrhage. Gastroenterology. 2011;141(1):62-70.

5. Crooks CJ, Card TR, West J. Excess Long-Term Mortality following NonVariceal Upper Gastrointestinal Bleeding: A Population-Based Cohort Study. PLoS Med. 2013;10(4):e1001437.

6. Nojkov B, Cappell MS. Distinctive aspects of peptic ulcer disease, Dieulafoy's lesion and Mallory-Weiss syndrome in patients with advanced alcoholic liver disease or cirrhosis. World J Gastroenterol. 2016;22(1):446.

7. Lee HJ, Han YM, Kim EH, Kim YJ, Hahm KB. A possible involvement of Nrf2mediated heme oxygenase-1 up-regulation in protective effect of the proton pump inhibitor pantoprazole against indomethacin-induced gastric damage in rats. BMC Gastroenterol. 2012;12(1):143.

8. Yang JX. Drug-drug Interaction in the Treatment of Gastrointestinal Tumors. Lat Am J Pharm. 2017;36(9):1784-8.

9. Tan $Q$, Joshua AM, Saggar JK, Yu M, Wang M, Kanga N, et al. Effect of pantoprazole to enhance activity of docetaxel against human tumour xenografts by inhibiting autophagy. Br J Cancer. 2015;112(5):832-40.

10. Tiberg F, Roberts J, Cervin C, Johnsson M, Sarp S, Tripathi AP, et al. Octreotide sc depot provides sustained octreotide bioavailability and similar IGF-1 suppression to octreotide LAR in healthy volunteers. $\mathrm{Br} \mathrm{J}$ Clin Pharmacol. 2015;80(3):460-72.

11. Monteiro S, Gonçalves TC, Magalhães J, Cotter J. Upper gastrointestinal bleeding risk scores: Who, when and why?. World J Gastrointest Pathophysiol. 2016;7(1):86-96.

12. Wang CY, Qin J, Wang J, Sun CY, Cao T, Zhu DD. Rockall score in predicting outcomes of elderly patients with acute upper gastrointestinal bleeding. World J Gastroenterol. 2013;19(22):3466-72.

Cite this article: Wang Y, Deng F. Clinical Effects of Pantoprazole and Octreotide in Upper Gastrointestinal Hemorrhage. Indian J of Pharmaceutical Education and Research. 2019;53(2):330-3. 